\title{
THERMAL CONVECTION OF RIVLIN-ERICKSEN FLUID IN THE PRESENCE OF VERTICAL ROTATION
}

\author{
A.S. Banyal ${ }^{{ }^{*}}$ and D.K. Sharma ${ }^{2}$ \\ ${ }^{1}$ Department of Mathematics, \\ Govt. College Nadaun, Dist. Hamirpur, (HP) INDIA 177033 \\ E mail: ajaibbanyal@ rediffmail.com \\ ${ }^{2}$ Department of Mathematics, \\ Rajiv Gandhi G. C. Kotshera, Shimla (HP), INDIA 171004
}

\begin{abstract}
The thermal instability of a Rivlin-Ericksen viscoelastic fluid, acted upon by uniform vertical rotation and heated from below, is investigated. Following linearized stability theory and normal mode analysis, the mathematical analysis of the governing equations of Rivlin-Ericksen viscoelastic fluid convection with a uniform vertical rotation is performed. It is shown that for the cases of rigid boundaries the complex growth rate $\sigma$ of oscillatory perturbations, neutral or unstable for all wave numbers, must lie inside a semi-circle, in the right-hand half of a complex $\sigma$-plane with the center at the origin. This prescribes the upper limits to the complex growth rate of arbitrary oscillatory motions of growing amplitude in a rotatory Rivlin-Ericksen viscoelastic fluid heated from below. Furthermore, the conditions necessary for the existence of oscillatory motions of growing amplitude in the present configuration and the sufficient condition for the validity of the Principle of Exchange of Stabilities are established.
\end{abstract}

Keywords: Thermal convection; Rivlin-Ericksen Fluid; rotation; PES; Rayleigh number; Taylor number.

\section{INTRODUCTION}

The stability of a dynamical system is closest to real life, in the sense that the realization of a dynamical system depends upon its stability. From the beginnings of conceptualizations of turbulence, the instability of fluid flows has been regarded as fundamental. The thermal instability of a fluid layer, which has an adverse temperature gradient that is maintained by heating from below, has an important role in Geophysics, the interior of the Earth, Oceanography, and Atmospheric Physics, and it has been investigated by several authors (Al- Doori, 2011; Bénard, 1900; Rayleigh, 1916; Jeffreys, 1926; Rana \& Thakur, 2012; Satya Narayana, Ramireddy, \& Venkataramana, 2011) under different conditions. A detailed account of the theoretical and experimental study of the onset of Bénard Convection in Newtonian fluids, under varying assumptions of hydrodynamics and hydromagnetics, has been given by Chandrasekhar (1981). The use of the Boussinesq approximation has been made throughout, which states that density changes are disregarded in all other terms of the equation of motion, except the external force term. Bhatia and Steiner (1972) have considered the effect of uniform rotation on the thermal instability of a viscoelastic (Maxwell) fluid, and they found that rotation has a destabilizing influence in contrast to the stabilizing effect it has on a Newtonian fluid. The thermal instability of a Maxwell fluid in hydromagnetics has been studied by Bhatia and Steiner (1973). They found that the magnetic field stabilizes 
a viscoelastic (Maxwell) fluid just as it does a Newtonian fluid. Sharma (1976) studied the thermal instability of a layer of viscoelastic (Oldroydian) fluid acted upon by a uniform rotation and found that rotation has destabilizing as well as stabilizing effects under certain conditions, in contrast to that of a Maxwell fluid, in which it has a destabilizing effect. In another study, Sharma (1975) investigated the stability of a layer of an electrically conducting Oldroyd (1958) fluid in the presence of a magnetic field, and found that the magnetic field has a stabilizing influence.

Many elastico-viscous fluids cannot be characterized by Maxwell's constitutive relations or by Oldroyd's (1958) constitutive relations. Two such classes of fluids are Rivlin-Ericksen's and Walter's (model B') fluids. Rivlin-Ericksen (1955) proposed a theoretical model for one such class of elastico-viscous fluids. Sharma and Kumar (1996) studied the effect of rotation on the thermal instability of a Rivlin-Ericksen elastico-viscous fluid, and found that rotation has a stabilizing effect and that it introduces oscillatory modes in the skystem. Kumar, Mohan, and Lal (2006) considered effects of rotation and magnetic field with free boundaries only, on a Rivlin-Ericksen elastico-viscous fluid, and found that rotation has a stabilizing effect, whereas a magnetic field has both stabilizing and destabilizing effects. A layer of such fluid, heated from below or under the action of a magnetic field or rotation or both, may find applications in Geophysics, interior of the Earth, Oceanography, and Atmospheric Physics.

Pellow and Southwell (1940) proved the validity of the Principle of Exchange of Stabilities for the classical Rayleigh-Bénard convection problem. Banerjee, Katoch, Dube, and Banerjee (1981) presented a new scheme for combining the governing equations of thermohaline convection. This is shown to lead to the bounds for the complex growth rate of the arbitrary oscillatory perturbations, neutral or unstable for all combinations of dynamically rigid or free boundaries. In addition, Banerjee and Banerjee (1984) established a criterion for the characterization of non-oscillatory motions in hydrodynamics, which was further extended by Gupta, Sood, and Bhardwaj (1986). However, no such result exists for non-Newtonian fluid configurations, in general and for Rivlin-Ericksen viscoelastic fluid configurations, in particular. Banyal (2011) characterized the non-oscillatory motions in a couple-stress fluid. Bearing in mind the importance of non-Newtonian fluids, the present paper attempts to prescribe the upper limits to the complex growth rate of arbitrary oscillatory motions of growing amplitude in a layer of incompressible Rivlin-Ericksen viscoelastic fluid, which is heated from below in the presence of uniform vertical rotation, opposite to force of gravity, when the bounding surfaces at the top and bottom of the fluid are rigid with infinite horizontal extension.

\section{FORMULATION OF THE PROBLEM AND PERTURBATION EQUATIONS}

Consider an infinite horizontal incompressible Rivlin-Ericksen viscoelastic fluid layer of thickness $d$, heated from below, such that the temperature and density at the bottom surface $z=0$, are $T_{0}$ and $\rho_{0}$ and at the upper surface $z=d$, are $T_{d}$ and $\rho_{d}$, respectively, and that a uniform adverse temperature gradient $\beta\left(=\left|\frac{d T}{d z}\right|\right)$ is maintained. The fluid is acted upon by a uniform vertical rotation $\vec{\Omega}(0,0, \Omega)$ parallel to the force field of gravity $\vec{g}(0,0,-g)$. 
The equations of motion, continuity, and heat conduction, governing the flow of the Rivlin-Ericksen viscoelastic fluid in the presence of rotation are (Rivlin \& Ericksen, 1955; Chandrasekhar, 1981; Kumar et al., 2006):

$$
\begin{gathered}
\frac{\partial \vec{q}}{\partial t}+(\vec{q} \cdot \nabla) \vec{q}=-\nabla\left(\frac{p}{\rho_{o}}-\frac{1}{2}|\vec{\Omega} \times \vec{r}|^{2}\right)+\vec{g}\left(1+\frac{\delta \rho}{\rho_{0}}\right)+\left(v+v^{\prime} \frac{\partial}{\partial t}\right) \nabla^{2} \vec{q}+2(\vec{q} \times \vec{\Omega}) \\
\nabla \cdot \vec{q}=0 \\
\frac{\partial T}{\partial t}+(\vec{q} \cdot \nabla) T=\kappa \nabla^{2} T
\end{gathered}
$$

where $\rho, p, T, v, v^{\prime}$, and $\vec{q}(u, v, w)$ denote the density, pressure, temperature, kinematic viscosity, kinematic viscoelasticity, and velocity of the fluid, respectively, and where $\vec{r}(x, y, z)$.

The equation of state for the fluid is

$$
\rho=\rho_{0}\left[1-\alpha\left(T-T_{0}\right)\right]
$$

where the suffix zero refers to the values at the reference level $z=0$. Here, $\vec{g}(0,0,-g)$ is the acceleration due to gravity and $\alpha$ is the coefficient of thermal expansion. In writing Eq. (1), we made use of the Boussinesq approximation, which states that the density variations are ignored in all terms of the equation of motion, except the external force term. The thermal diffusivity $\kappa$ is assumed constant.

The initial state is one in which the velocity, density, pressure, and temperature at any point in the fluid are given by

$$
\vec{q}=(0,0,0), \rho=\rho(z), p=p(\mathrm{z}), T=T(\mathrm{z})
$$

Assume small perturbations around the basic solution and let $\delta \rho, \delta p, \theta$, and $\vec{q}(u, v, w)$ denote the perturbations in density $\rho$, pressure $p$, temperature $T$ and velocity $\vec{q}(0,0,0)$, respectively. The change in density $\delta \rho$, caused mainly by the perturbation $\theta$ in temperature, is given by

$$
\rho+\delta \rho=\rho_{0}\left[1-\alpha\left(T+\theta-T_{0}\right)\right]=\rho-\alpha \rho_{0} \theta, \text { i.e. } \delta \rho=-\alpha \rho_{0} \theta
$$

Then, the linearized perturbation equations are:

$$
\begin{gathered}
\frac{\partial \vec{q}}{\partial t}=-\frac{1}{\rho_{0}} \nabla \delta p-\vec{g} \alpha \theta+\left(v+v^{\prime} \frac{\partial}{\partial t}\right) \nabla^{2} \vec{q}+2(\vec{q} \times \vec{\Omega}) \\
\nabla \cdot \vec{q}=0 \\
\frac{\partial \theta}{\partial t}=\beta w+\kappa \nabla^{2} \theta
\end{gathered}
$$

Within the framework of the Boussinesq approximation, Eqs. (6)-(9) become:

$$
\frac{\partial}{\partial t} \nabla^{2} w=\left(v+v^{\prime} \frac{\partial}{\partial t}\right) \nabla^{4} w+g \alpha\left(\frac{\partial^{2} \theta}{\partial x^{2}}+\frac{\partial^{2} \theta}{\partial y^{2}}\right)-2 \Omega \frac{\partial \varsigma}{\partial z}
$$




$$
\begin{gathered}
\frac{\partial \varsigma}{\partial t}=\left(v+v^{\prime} \frac{\partial}{\partial t}\right) \nabla^{2} \varsigma+2 \Omega \frac{\partial w}{\partial z}, \\
\frac{\partial \theta}{\partial t}=\beta w+\kappa \nabla^{2} \theta
\end{gathered}
$$

where $\nabla^{2}=\frac{\partial^{2}}{\partial x^{2}}+\frac{\partial^{2}}{\partial y^{2}}+\frac{\partial^{2}}{\partial z^{2}}$ and $\varsigma=\frac{\partial v}{\partial x}-\frac{\partial u}{\partial y}$ denote the $z$-component of vorticity.

\section{NORMAL MODE ANALYSIS}

Analyzing the disturbances into normal modes, we assume that the perturbation quantities are of the form:

$$
[w, \theta, \varsigma]=[W(z), \Theta(z), Z(z)] \exp \left(i k_{x} x+i k_{y} y+n t\right),
$$

where $k_{x}, k_{y}$ are the wave numbers along the $x$ - and $y$-directions, respectively, $k=\left(k_{x}^{2}+k_{y}^{2}\right)^{\frac{1}{2}}$ is the resultant wave number, and $n$ is the growth rate that in general, is a complex constant.

Using Eq. (13), Eqs. (10)-(12), in non-dimensional form, transform to:

$$
\begin{gathered}
\left.\left(D^{2}-a^{2}\right)(1+F \sigma)\left(D^{2}-a^{2}\right)-\sigma\right] W=R a^{2} \Theta+T_{A} D Z \\
{\left[(1+F \sigma)\left(D^{2}-a^{2}\right)-\sigma\right] Z=-D W} \\
\left(D^{2}-a^{2}-p_{1} \sigma\right) \Theta=-W
\end{gathered}
$$

where we have introduced new coordinates $\left(x^{\prime}, y^{\prime}, z^{\prime}\right)=(x / d, y / d, z / d)$ in new units of length $d$ and $D=d / d z^{\prime}$. For convenience, the dashes are dropped hereafter. In addition, we have substituted $a=k d, \sigma=\frac{n d^{2}}{v}, p_{1}=\frac{v}{\kappa}$, which is the thermal Prandtl number; $\quad p_{2}=\frac{v}{\eta}$ is the magnetic Prandtl number, $F=\frac{v^{\prime}}{d^{2}}$ is the Rivlin-Ericksen kinematic viscoelasticity parameter, $R=\frac{g \alpha \beta d^{4}}{\kappa v}$ is the thermal Rayleigh number, and $T_{A}=\frac{4 \Omega^{2} d^{4}}{v^{2}}$ is the Taylor number. Furthermore, we have substituted $W=W_{\oplus}$, $\Theta=\frac{\beta d^{2}}{\kappa} \Theta_{\oplus}, Z=\frac{2 \Omega d}{v} Z_{\oplus}$, and $D_{\oplus}=d D$, and dropped $(\oplus)$ for convenience.

We now consider the case where both boundaries are rigid, perfectly conducting and maintained at constant temperature. Thus, the perturbations in temperature at the boundaries are zero. The appropriate boundary conditions with respect to which Eqs. (14)-(16), must possess a solution are:

$$
\mathrm{W}=\mathrm{DW}=0, \Theta=0 \text { and } \mathrm{Z}=0 \text { at } \mathrm{z}=0 \text { and } \mathrm{z}=1 \text {. }
$$

Eqs. (14)-(16), together with boundary conditions (17), pose an eigenvalue problem for $\sigma$ and we wish to characterize $\sigma_{i}$ when $\sigma_{r} \geq 0$. 
We first note that $W$ and $Z$ satisfy $W(0)=0=W(1)$ and $Z(0)=0=Z(1)$ in addition to satisfying the governing equations and hence, we have from the Rayleigh-Ritz inequality (Schultz, 1973)

$$
\int_{0}^{1}|D W|^{2} d z \geq \pi^{2} \int_{0}^{1}|W|^{2} d z
$$

and

$$
\int_{0}^{1}|D Z|^{2} d z \geq \pi^{2} \int_{0}^{1}|Z|^{2} d z
$$

Furthermore, for $W(0)=0=W(1)$ and $Z(0)=0=Z(1)$, Banerjee, Gupta \& Prakash (1993) have shown that

$$
\int_{0}^{1}\left|D^{2} W\right|^{2} d z \geq \pi^{2} \int_{0}^{1}|D W|^{2} d z \text { And } \int_{0}^{1}\left|D^{2} Z\right|^{2} d z \geq \pi^{2} \int_{0}^{1}|D Z|^{2} d z
$$

\section{MATHEMATICAL ANALYSIS}

We prove the following lemma:

Lemma 1: For any arbitrary oscillatory perturbation, neutral or unstable:

$$
\int_{0}^{1}|Z|^{2} d z\left\langle\frac { 1 } { \pi ^ { 4 } } \int _ { 0 } ^ { 1 } | D W | ^ { 2 } d z \text { and } \int _ { 0 } ^ { 1 } \{ D Z | ^ { 2 } + a ^ { 2 } | Z | ^ { 2 } \} d z \left\langle\frac{1}{\pi^{2} F^{2}|\sigma|^{2}} \int_{0}^{1}|D W|^{2} d z\right.\right. \text {. }
$$

Proof: Multiplying Eq. (15) with its complex conjugate and integrating by parts, for an appropriate number of times, each term on both sides of the resulting equation and by making use of boundary condition on $Z$, namely $Z(0)=0=Z(1)$, together with Eq. (17), we get:

$$
\begin{aligned}
& \left(1+2 F \sigma_{r}+F^{2}|\sigma|^{2}\right) \int_{0}^{1}\left\{\left|D^{2} Z\right|^{2}+2 a^{2}|D Z|^{2}+a^{4}|Z|^{2}\right\} d z+\left(2 \sigma_{r}\right. \\
& \left.+2 F|\sigma|^{2}\right) \int_{0}^{1}\left\{\left.D Z\right|^{2}+a^{2}|Z|^{2}\right\} d z++|\sigma|^{2} \int_{0}^{1}|Z|^{2} d z=\int_{0}^{1}|D W|^{2} d z
\end{aligned}
$$

On utilizing the inequalities Eqs. (19) and (20), Eq. (21) gives:

$$
\int_{0}^{1}|Z|^{2} d z\left\langle\frac { 1 } { \pi ^ { 4 } } \int _ { 0 } ^ { 1 } | D W | ^ { 2 } d z \text { and } \int _ { 0 } ^ { 1 } \{ | D Z | ^ { 2 } + a ^ { 2 } | Z | ^ { 2 } \} d z \left\langle\frac{1}{\pi^{2} F^{2}|\sigma|^{2}} \int_{0}^{1}|D W|^{2} d z\right.\right.
$$

We prove the following theorem:

Theorem 1: If $\mathrm{R}>0, \mathrm{~F}>0, T_{A}>0, \sigma_{r} \geq 0$, and $\sigma_{i} \neq 0$, then the necessary condition for the existence of non-trivial solution $(W, \Theta, Z)$ of Eqs. (16)-(18), together with the boundary conditions of Eq. (19), is that 


$$
|\sigma|^{2}\left\langle\frac{T_{A}}{\pi^{2} F\left(1+\pi^{2} F\right)\left\{1-\frac{T_{A}}{\pi^{4}\left(1+\pi^{2} F\right)}\right\}} .\right.
$$

Proof: Multiplying Eq. (14) by $W^{*}$ (the complex conjugate of W) throughout and by integrating the resulting equation over the vertical range of $z$, we get

$$
(1+F \sigma) \int_{0}^{1} W^{*}\left(D^{2}-a^{2}\right)^{2} W d z-\sigma \int_{0}^{1} W^{*}\left(D^{2}-a^{2}\right) W d z=R a^{2} \int_{0}^{1} W^{*} \Theta d z+T_{A} \int_{0}^{1} W^{*} D Z d z .
$$

Taking the complex conjugate on both sides of Eq. (16), we get

$$
\left(D^{2}-a^{2}-p_{1} \sigma^{*}\right) \Theta^{*}=-W^{*}
$$

Therefore, by using (24), we get

$$
\int_{0}^{1} W^{*} \Theta d z=-\int_{0}^{1} \Theta\left(D^{2}-a^{2}-p_{1} \sigma^{*}\right) \Theta^{*} d z
$$

Furthermore, by taking the complex conjugate on both sides of Eq. (15), we get

$$
\left\lfloor\left(1+F \sigma^{*}\right)\left(D^{2}-a^{2}\right)-\sigma^{*}\right\rfloor Z^{*}=-D W^{*} .
$$

Therefore, by using Eq. (26) and the appropriate boundary conditions of Eq. (17), we get

$$
\int_{0}^{1} W^{*} D Z d z=-\int_{0}^{1} D W^{*} Z d z=\int_{0}^{1} Z\left\{\left(1+F \sigma^{*}\right)\left(D^{2}-a^{2}\right)-\sigma^{*}\right\} Z^{*} d z
$$

Substituting Eqs. (25) and (27) in the right-hand side of Eq. (23), we get

$$
\begin{gathered}
(1+F \sigma) \int_{0}^{1} W^{*}\left(D^{2}-a^{2}\right)^{2} W d z-\sigma \int_{0}^{1} W^{*}\left(D^{2}-a^{2}\right) W d z \\
=-R a^{2} \int_{0}^{1} \Theta\left(D^{2}-a^{2}-p_{1} \sigma^{*} \Theta^{*} d z+T_{A} \int_{0}^{1} Z\left\{\left(1+F \sigma^{*}\right)\left(D^{2}-a^{2}\right)-\sigma^{*}\right\} Z^{*} d z .\right.
\end{gathered}
$$

Integrating the terms on both sides of Eq. (25) for an appropriate number of times, by making use of the appropriate boundary conditions of Eq. (19), together with Eq. (17), we get

$$
\begin{gathered}
(1+F \sigma) \int_{0}^{1}\left\{\left.D^{2} W\right|^{2}+2 a^{2}|D W|^{2}+a^{4}|W|^{2}\right\} d z+\sigma \int_{0}^{1}\left\{\left.D W\right|^{2}+a^{2}|W|^{2}\right\} d z \\
=R a^{2} \int_{0}^{1}\left\{|D \Theta|^{2}+a^{2}|\Theta|^{2}+p_{1} \sigma^{*}|\Theta|^{2}\right\} d z-T_{A}\left(1+F \sigma^{*}\right) \int_{0}^{1}\left\{|D Z|^{2}+a^{2}|Z|^{2}\right\} d z-T_{A} \sigma^{*} \int_{0}^{1}|Z|^{2} d z .
\end{gathered}
$$

Then, by equating the imaginary parts on both sides of Eq. (29) and cancelling $\sigma_{i}(\neq 0)$ throughout, we get

$$
\begin{aligned}
& F \int_{0}^{1}\left\{\left.D^{2} W\right|^{2}+2 a^{2}|D W|^{2}+a^{4}|W|^{2}\right\} d z+\int_{0}^{1}\left\{|D W|^{2}+a^{2}|W|^{2}\right\} d z \\
& \quad=-R a^{2} p_{1} \int_{0}^{1}|\Theta|^{2} d z+T_{A} F \int_{0}^{1}\left\{|D Z|^{2}+a^{2}|Z|^{2}\right\} d z+T_{A} \int_{0}^{1}|Z|^{2} d z
\end{aligned}
$$


Now, $\mathrm{R}>0, \mathrm{~F}>0$, and $T_{A}>0$ and by utilizing the inequalities of Eqs. (19), (20), and (22), Eq. (30) gives

$$
\left[\left(1+\pi^{2} F\right)-\left\{\frac{T_{A}}{\pi^{2} F|\sigma|^{2}}+\frac{T_{A}}{\pi^{4}}\right\}\right]_{0}^{1}|D W|^{2} d z+I_{1}\langle 0,
$$

where

$$
I_{1}=F \int_{0}^{1}\left\{2 a^{2}|D W|^{2}+a^{4}|W|^{2}\right\} d z+a^{2} \int_{0}^{1}|W|^{2} d z+R a^{2} p_{1} \int_{0}^{1}|\Theta|^{2} d z,
$$

is positive definite.

Therefore, we must have

$$
|\sigma|^{2}\left\langle\frac{T_{A}}{\pi^{2} F\left(1+\pi^{2} F\right)\left\{1-\frac{T_{A}}{\pi^{4}\left(1+\pi^{2} F\right)}\right\}} .\right.
$$

Hence, if

$$
\sigma_{r} \geq 0 \text { and } \sigma_{i} \neq 0, \text { then }|\sigma|^{2}\left\langle\frac{T_{A}}{\pi^{2} F\left(1+\pi^{2} F\right)\left\{1-\frac{T_{A}}{\pi^{4}\left(1+\pi^{2} F\right)}\right\}}\right.
$$

and this completes the proof of the theorem.

In the context of the existence of instability in 'oscillatory modes' and that of 'overstability' in the present configuration, we can state prove a theorem as follows.

Theorem 2: The necessary condition for the existence of instability in 'oscillatory modes' and that of 'overstability' in a Rivlin-Ericksen viscoelastic fluid, heated from below in the presence of uniform vertical rotation, is that the Taylor number $T_{A}$ and the viscoelasticity parameter of the fluid $F$, must satisfy the inequality $\frac{T_{A}}{\pi^{4}\left(1+\pi^{2} F\right)}\langle 1$, when both the bounding surfaces are rigid.

Proof: The inequality Eq. (32) for $\sigma_{r} \geq 0$ and $\sigma_{i} \neq 0$, can be written as:

$$
\sigma_{r}^{2}+\sigma_{i}^{2}\left\langle\frac{T_{A}}{\pi^{2} F\left(1+\pi^{2} F\right)\left\{1-\frac{T_{A}}{\pi^{4}\left(1+\pi^{2} F\right)}\right\}}\right. \text {. }
$$

We necessarily have $\frac{T_{A}}{\pi^{4}\left(1+\pi^{2} F\right)}\langle 1$,

which completes the proof.

Presented otherwise, from the point of view of the existence of instability as stationary convection, the above theorem can be put in the form as follows.

Theorem 3: The sufficient condition for the validity of the 'exchange principle' and the onset of instability as non-oscillatory motions of non-growing amplitude in a Rivlin- 
Ericksen viscoelastic fluid, heated from below in the presence of uniform vertical rotation, is that $\left.\frac{T_{A}}{\pi^{4}\left(1+\pi^{2} F\right)}\right\rangle 1$, where $T_{A}$ is the Taylor number and $F$ is the viscoelasticity parameter, when both bounding surfaces are rigid.

Or, the onset of instability in a Rivlin-Ericksen viscoelastic fluid, heated from below in the presence of uniform vertical rotation, cannot manifest itself as oscillatory motions of growing amplitude if the Taylor number $T_{A}$ and the viscoelasticity parameter $F$, satisfy the inequality $\left.\frac{T_{A}}{\pi^{4}\left(1+\pi^{2} F\right)}\right\rangle 1$, when both bounding surfaces are rigid.

Theorem 4: For stationary convection, the Rivlin-Ericksen viscoelastic fluid behaves like an ordinary Newtonian fluid, i.e., $T_{A}=0$ implies that $\sigma_{r}=0$ and $\sigma_{i}=0$, when both bounding surfaces are rigid.

Proof: The inequality Eq. (32), can be written as:

$$
\sigma_{r}^{2}+\sigma_{i}^{2}\left\langle\frac{T_{A}}{\pi^{2} F\left(1+\pi^{2} F\right)\left\{1-\frac{T_{A}}{\pi^{4}\left(1+\pi^{2} F\right)}\right\}}\right. \text {. }
$$

If $T_{A}=0$, then we necessarily have $\sigma_{r}=0$ and $\sigma_{i}=0$. Thus, for stationary convection, the Rivlin-Ericksen viscoelastic fluid behaves like an ordinary Newtonian fluid, when both the bounding surfaces are rigid and it mathematically establishes the result of Kumar et al. (2006). This completes the proof.

\section{CONCLUSION}

The inequality Eq. (32) for $\sigma_{r} \geq 0$ and $\sigma_{i} \neq 0$, can be written as:

$$
\sigma_{r}^{2}+\sigma_{i}^{2}\left\langle\frac{T_{A}}{\pi^{2} F\left(1+\pi^{2} F\right)\left\{1-\frac{T_{A}}{\pi^{4}\left(1+\pi^{2} F\right)}\right\}}\right. \text {. }
$$

The essential content of the theorem, from the point of view of linear stability theory, is that for the configuration of a Rivlin-Ericksen viscoelastic fluid of infinite horizontal extension, heated from below in the presence of uniform vertical rotation parallel to the force field of gravity, and having rigid top and bottom bounding surfaces, the complex growth rate of arbitrary oscillatory motions of growing amplitude, must lie inside a semi-circle in the right-hand half of the $\sigma_{r} \sigma_{i}$ - plane, whose center is at the origin and whose radius is $\sqrt{\frac{\pi^{2} T_{A}}{F\left\{\pi^{4}\left(1+\pi^{2} F\right)-T_{A}\right\}}}$, where $T_{A}$ is the Taylor number and $F$ is the viscoelasticity parameter.

Furthermore, it follows from inequality Eq. (32) that a sufficient condition for the validity of the Principle of Exchange of Stabilities in rotatory Rivlin-Ericksen viscoelastic fluid convection is that $\left.\frac{T_{A}}{\pi^{4}\left(1+\pi^{2} F\right)}\right\rangle 1$. It is therefore clear that the 
existence of oscillatory motions of growing amplitude in the present configuration depends crucially upon the magnitude of the non-dimensional number $\frac{\pi^{4}\left(1+\pi^{2} F\right)}{T_{A}}$, in the sense that as long as $0<\frac{\pi^{4}\left(1+\pi^{2} F\right)}{T_{A}} \leq 1$, no such motions are possible, and in particular, the Principle of Exchange of Stabilities is valid and provides significant improvement to Banyal (2012).

\section{ACKNOWLEDGMENTS}

The authors are highly thankful to the referees for their very constructive, valuable suggestions and useful technical comments, which led to a significant improvement of the paper.

\section{REFERENCES}

Al- Doori, W. H. A. R. (2011). Enhancement of natural convection heat transfer from the rectangular fins by circular perforations. International Journal of Automotive and Mechanical Engineering, 4, 428-436.

Banerjee, M. B., Katoch, D. C., Dube, G. S., \& Banerjee, K. (1981). Bounds for growth rate of perturbation in thermohaline convection. Proceedings of the Royal Society A: Mathematical, Physical and Engineering Sciences, 378, 301-304.

Banerjee, M. B., \& Banerjee, B. (1984). A characterization of non-oscillatory motions in magnetohydronamics. Indian Journal of Pure and Applied Mathematics, 15(4), 377-382.

Banerjee, M. B., Gupta, J. R., \& Prakash, J. (1993). On thermohaline convection of Veronis type. Journal of Mathematical Analysis and Applications, 179, 327-334.

Banyal, A. S. (2011). The necessary condition for the onset of stationary convection in couple-stress fluid. International Journal of Fluid Mechanics Research, 38(5), 450-457.

Banyal, A. S. (2012). Upper limit to the growth rate of perturbations in rivlin-ericksen viscoelastic fluid in the presence of rotation. International Journal of Advances in Engineering Research, 3, 33-42.

Bénard, H. (1900). Les tourbillions cellulaires dans une nappe liquid. Revue Genérale des Sciences Pures et Appliquees, 11, 1309-1328.

Bhatia, P. K., \& Steiner, J. M. (1972). Convective instability in a rotating viscoelastic fluid layer. Zeitschrift fur Angewandte Mathematik and Mechanik, 52, 321-327.

Bhatia, P. K., \& Steiner, J. M. (1973). Thermal instability in a viscoelastic fluid layer in hydromagnetics. Journal of Mathematical Analysis and Applications, 41, 271283.

Chandrasekhar, S. (1981). Hydrodynamic and hydromagnetic stability. New York: Dover Publication.

Gupta, J. R., Sood, S. K., \& Bhardwaj, U. D. (1986). On the characterization of nonoscillatory motions in rotatory hydromagnetic thermohaline convection. Indian Journal of Pure and Applied Mathematics, 17(1),100-107.

Jeffreys, H. (1926). The stability of a fluid layer heated from below. Philosophical Magazine, 2, 833-844. 
Kumar, P., Mohan, H., \& Lal, R. (2006). Effect of magnetic field on thermal instability of a rotating Rivlin-Ericksen viscoelastic fluid. International Journal of Mathematics and Mathematical Sciences, 2006(28042), 1-10.

Oldroyd, J. G. (1958). Non-Newtonian effects in steady motion of some idealized elastic-viscous liquids. Proceedings of the Royal Society of London A, 245, 278297.

Pellow, A., \& Southwell, R. V. (1940). On the maintained convective motion in a fluid heated from below. Proceedings of the Royal Society A: Mathematical, Physical and Engineering Sciences, 176, 312-43.

Rana, G. C., \& Thakur, R. C. (2012). Effect of suspended particles on thermal convection in Rivlin-Ericksen fluid in A Darcy-Brinkman porous medium. Journal of Mechanical Engineering and Sciences, 2, 162-171.

Rayleigh, L. (1916). On convective currents in a horizontal layer of fluid when the higher temperature is on the underside. Philosophical Magazine, 32, 529-546.

Rivlin, R. S., \& Ericksen, J. L. (1955). Stress deformation relations for isotropic materials. Journal of Rational Mechanical Analysis, 4, 323-429.

Satya Narayana, P. V., Ramireddy, G., \& Venkataramana, S. (2011). Hall current effects on free-convection MHD flow past a porous plate. International Journal of Automotive and Mechanical Engineering, 3, 350-363.

Schultz, M. H. (1973). Spline analysis, prentice hall. New Jersy: Englewood Cliffs.

Sharma, R. C. (1975). Thermal instability in a viscoelastic fluid in hydromagnetics. Acta Physica Hungarica, 38, 293-298.

Sharma, R. C. (1976). Effect of rotation on thermal instability of a viscoelastic fluid. Acta Physica Hungarica, 40, 11-17.

Sharma, R. C., \& Kumar, P. (1996). Effect of rotation on thermal instability in RivlinEricksen elastico-viscous fluid. Zeitschrift fur Naturforschung, 51, 821-824. 